\title{
Formaldehyde as a carbon and electron shuttle between autotroph and heterotroph populations in acidic hydrothermal vents of Norris Geyser Basin, Yellowstone National Park
}

\author{
Authors: James J. Moran, Laura M. Whitmore, Nancy G. \\ Isern, Margaret F. Romine, Krystin M. Riha, William P. \\ Inskeep, \& Helen W. Kreuzer
}

The final publication is available in Extremophiles at Springer via http://dx.doi.org/10.1007/ $\underline{\underline{s 00792-016-0821-2}}$

Moran, James J. , Laura M. Whitmore, Nancy G. Isern, Margaret F. Romine, Krystin M. Riha, William P. Inskeep, and Helen W. Kreuzer. "Formaldehyde as a carbon and electron shuttle between autotroph and heterotroph populations in acidic hydrothermal vents of Norris Geyser Basin, Yellowstone National Park." Extremophiles 20, no. 3 (May 2016): 291-299.

DOI: $10.1007 / \mathrm{s} 00792-016-0821-2$.

Made available through Montana State University's $\underline{\text { ScholarWorks }}$ scholarworks. montana.edu 


\title{
Formaldehyde as a carbon and electron shuttle between autotroph and heterotroph populations in acidic hydrothermal vents of Norris Geyser Basin, Yellowstone National Park
}

\author{
James J. Moran' ${ }^{1}$ Laura M. Whitmore ${ }^{1,5} \cdot$ Nancy G. Isern ${ }^{2} \cdot$ Margaret F. Romine $^{3}$. \\ Krystin M. Riha ${ }^{1} \cdot$ William P. Inskeep $^{4} \cdot$ Helen W. Kreuzer ${ }^{1}$ \\ Chemical and Biological Signature Science Group, Pacific Northwest National Laboratory, Richland, WA, USA \\ Environmental and Molecular Science Laboratory, Pacific Northwest National Laboratory, Richland, WA, USA \\ Microbiology Department, Pacific Northwest National Laboratory, Richland, WA, USA \\ Department of Land Resources and Environmental Sciences, Montana State University, Bozeman, MT, USA \\ Present Address: Department of Marine Science, University of Southern Mississippi, Stennis Space Center, MS 39529, USA
}

\begin{abstract}
The Norris Geyser Basin in Yellowstone National Park contains a large number of hydrothermal systems, which host microbial populations supported by primary productivity associated with a suite of chemolitho-trophic metabolisms. We demonstrate that Metallosphaera yellowstonensis MK1, a facultative autotrophic archaeon isolated from a hyperthermal acidic hydrous ferric oxide (HFO) spring in Norris Geyser Basin, excretes formalde-hyde during autotrophic growth. To determine the fate of formaldehyde in this low organic carbon environment, we incubated native microbial mat (containing M. yellowston-ensis) from a HFO spring with ${ }^{13} \mathrm{C}$-formaldehyde. Isotopic analysis of incubation-derived $\mathrm{CO} 2$ and biomass showed that formaldehyde was both oxidized and assimilated by members of the community. Autotrophy, formaldehyde oxidation, and formaldehyde assimilation displayed different sensitivities to chemical inhibitors, suggesting that distinct subpopulations in the mat selectively perform these functions. Our results demonstrate that electrons originally resulting from iron oxidation can energetically fuel auto-trophic carbon fixation and associated formaldehyde excretion, and that formaldehyde is both oxidized and assimi-lated by different organisms within the native microbial community. Thus, formaldehyde can effectively act as a carbon and electron shuttle connecting the autotrophic, iron oxidizing members with associated heterotrophic members in the HFO community.
\end{abstract}

Keywords Acidophilic $\cdot$ Carbon transfer $\cdot$ Yellowstone National Park $\cdot$ Archaea $\cdot$ Carbon cycle

\section{Introduction}

Norris Geyser Basin in Yellowstone National Park (YNP), Wyoming, USA, is home to a high density of hydrothermal springs. The high temperatures $\left(>65^{\circ} \mathrm{C}\right)$ and extreme acidic conditions $(\mathrm{pH} \sim 3)$ in many of these systems preclude photosynthetic autotrophy (Boyd et al. 2009). The abundant (up to $60 \mu \mathrm{M}$ ) ferrous iron content of these systems, however, provides an energy source for chemolithoautotrophy using iron oxidation, since the equivalent abiotic reaction at these low pH values is kinetically hindered (Inskeep et al. 2005; Kozubal et al. 2012; Singer and Stumm 1970). The resulting hydrous ferric oxide (HFO) microbial communi-ties contain a diverse group of different archaea including members of the Sulfolobales, Desulfurococcales, Thermo-proteales, Thaumarchaeota, and Geoarchaeota, and acidophilic bacteria from the Aquificales (Inskeep et al. 2010; Kozubal et al. 2012, 2013). A previously unrecognized 
Sulfolobales, Metallosphaera yellowstonensis MK1, was isolated from HFO mats of Norris Geyser Basin, YNP (Kozubal et al. 2008). Metagenomic analyses have detected MK1-like organisms in 11 iron mat communities within YNP and revealed that they can be a major component ( $10-20 \%$ of biomass) of the archaeal population in these systems (Kozubal et al. 2008, 2012).

Jennings et al. (Jennings et al. 2014) demonstrated that axenic M. yellowstonensis cultures autotrophically fix $\mathrm{CO}_{2}$ into biomass, likely through the 3-hydroxypropionate/4hydroxybutyrate pathway, when performing iron oxidation. Similarly, Jennings et al. (Jennings et al. 2014) used incubations of HFO mat from Norris Geyser Basin to demonstrate autotrophic $\mathrm{CO}_{2}$ fixation consistent with M. yellowstonensis activity and suggest a large portion of the mat's carbon originates from in situ autotrophic activity. Previous metagenome analysis suggests other archaeal organisms within the HFO mats are primarily heterotrophic (Beam et al. 2014; Kozubal et al. 2013), with predicted carbon sources originating from autotrophic organism (including Metallosphaera) metabolism and, to a lesser extent, from organics released from the surrounding landscape.

Exchange of fixed carbon between autotrophic and heterotrophic members of the HFO could occur via multiple mechanisms including consumption of dead biomass and secretion/uptake of metabolites. Here we present evidence that M. yellowstonensis secretes formaldehyde during autotrophic growth on ferrous iron and further demonstrate that organisms within the HFO mat communities both oxidize formaldehyde to $\mathrm{CO}_{2}$ and incorporate formaldehyde-derived carbon into mat biomass. Thus, in these communities, electrons can be taken from ferrous iron by M. yellowstonensis, used to fix $\mathrm{CO}_{2}$, and a portion of the electrons, as well as $\mathrm{C}$, passed to associated community member(s) via formaldehyde. In this manner, formaldehyde could serve as one possible electron and $\mathrm{C}$ shuttle within these archaeal-dominated communities.

\section{Materials and methods}

\section{Culturing M. yellowstonensis}

M. yellowstonensis strain MK1 was cultured at $65{ }^{\circ} \mathrm{C}$ in $50 \mathrm{~mL}$ artificial spring water medium in $125 \mathrm{~mL}$ glass bottles with septum-containing lids. The artificial spring water was formulated to mimic ion concentrations in the natural spring water and contained $400 \mu \mathrm{M} \mathrm{NaNO}, 2400 \mu \mathrm{M}$ $\mathrm{Na}_{2} \mathrm{SO}_{4}, 1200 \mu \mathrm{M} \mathrm{KCl}, 8000 \mu \mathrm{M} \mathrm{NaCL}, 114 \mu \mathrm{M}$ $\mathrm{AlCl}_{3} \cdot 6 \mathrm{H}_{2} \mathrm{O}, 717 \mu \mathrm{M} \mathrm{H}_{3} \mathrm{BO}_{3}, 12 \mu \mathrm{M} \mathrm{MgSO}{ }_{4}, 100 \mu \mathrm{M}$ $\mathrm{K}_{2} \mathrm{HPO}_{4}, 147 \mu \mathrm{M} \mathrm{KF}, 600 \mu \mathrm{M} \mathrm{NH} \mathrm{N}_{4} \mathrm{Cl}$, and $250 \mu \mathrm{M}$ $\mathrm{CaCl}_{2}$, with the $\mathrm{pH}$ adjusted to $2.5-3.0$ using $\mathrm{H}_{2} \mathrm{SO}_{4}$. To this medium we added (per liter) $1 \mathrm{~mL}$ of American Type Culture Collection (ATCC) Vitamin Solution MD-VS, and $1 \mathrm{~mL}$ ATCC Trace Mineral Stock Solution. The Trace Mineral Stock Solution contained $1.39 \mu \mathrm{M} \mathrm{ZnSO} \cdot 7 \mathrm{H}_{2} \mathrm{O}$, $5.01 \mu \mathrm{M} \mathrm{NiCl} \cdot 6 \mathrm{H}_{2} \mathrm{O}, 0.15 \mu \mathrm{M} \operatorname{VOSO}_{4} \cdot 2 \mathrm{H}_{2} \mathrm{O}, 2.5 \mu \mathrm{M}$ $\mathrm{Na}_{2} \mathrm{SeO}_{4}, 0.73 \mu \mathrm{M} \mathrm{MnCl} \cdot 4 \mathrm{H}_{2} \mathrm{O}, 0.3 \mu \mathrm{M} \mathrm{CuCl} \cdot 6 \mathrm{H}_{2} \mathrm{O}$, $0.1 \mu \mathrm{M} \mathrm{CoCl} \cdot 6 \mathrm{H}_{2} \mathrm{O}, 0.12 \mu \mathrm{M} \mathrm{Na}_{2} \mathrm{MoO}_{4} \cdot 2 \mathrm{H}_{2} \mathrm{O}$, and $0.3 \mu \mathrm{M} \mathrm{Na} \mathrm{WO}_{4} \cdot 2 \mathrm{H}_{2} \mathrm{O}$. Approximately $200 \mathrm{mg}$ of sterile pyrite was placed into each culture bottle to serve as an electron donor. Thirty $\mathrm{mL}$ (STP) of $\mathrm{CO}_{2}$ was injected into the headspace through the septum to provide an inorganic carbon source for autotrophy, and $30 \mathrm{~mL}$ (STP) $\mathrm{O}_{2}$ was injected to serve as an electron acceptor. Culture medium samples were extracted after 2 weeks of incubation and filtered through a $0.2 \mu \mathrm{m}$ filter to remove all particulates and biomass in preparation for analysis by nuclear magnetic resonance (NMR).

\section{Nuclear magnetic resonance analysis}

Proton-based NMR data were acquired on a Varian Direct Drive (VNMRS) $600 \mathrm{MHz}$ spectrometer (Agilent Technologies) equipped with a Dell Precision 390 Linux workstation running VNMRJ 2.2C. The spectrometer system was outfitted with a Varian triple resonance salt-tolerant cold probe with a cold carbon preamplifier. A Varian standard one-dimensional proton noesy with presaturation (tnnoesy) was collected on each sample, using a standard metabolomics data collection protocol: nonselective $90^{\circ}$ excitation pulse, $100 \mathrm{~ms}$ mixing time, acquisition time of $4 \mathrm{~s}$, recycle delay of $1 \mathrm{~s}$, spectral width of $12 \mathrm{ppm}$, and temperature control set to $25{ }^{\circ} \mathrm{C}$. Collected spectra were analyzed using Chenomx 7.1 software, with quantification based on spectral intensities relative to $0.5 \mathrm{mM}$ 2,2-dimethyl-2-silapentane-5-sulfonate, which was added as a spike to each sample.

\section{Ex situ microbial mat incubations}

We sampled HFO mat from "Beowulf Spring" $\left(44^{\circ} 43^{\prime}\right.$ $53.4^{\prime \prime} \mathrm{N}, 110^{\circ} 4240.9^{\prime \prime} \mathrm{W}$ ) within the Norris Geyser Basin, Yellowstone National Park, Wyoming, USA. The source water of Beowulf Spring $\left(80-84{ }^{\circ} \mathrm{C}\right)$ contains high levels of dissolved sulfide $(80-120 \mu \mathrm{M})$, which is oxidized to elemental $\mathrm{S}$ and deposited within the first $2-3 \mathrm{~m}$ as oxygen diffuses into the water channel (Inskeep et al. 2004; Langner et al. 2001). Samples of HFO microbial mats $\left(\sim 20 \mathrm{~cm}^{3}\right)$ were collected (3 May 2014) downstream of the S deposition zone ( $\sim 3-4 \mathrm{~m}$ from the source) at a temperature of $65-68{ }^{\circ} \mathrm{C}$ and added to $60 \mathrm{~mL}$ of spring water. Samples were maintained circa in situ spring temperature $\left(\sim 65^{\circ} \mathrm{C}\right)$ during transport to Pacific Northwest National Laboratory (Richland, WA) for experimental incubations. 
Table 1 Experimental incubation design

\begin{tabular}{|c|c|c|c|c|c|c|c|}
\hline \multirow[t]{2}{*}{ Experimental type } & \multirow[t]{2}{*}{ Experimental or control } & \multicolumn{2}{|c|}{$\begin{array}{l}\text { Headspace } \\
\text { additions (mL) }\end{array}$} & \multirow{2}{*}{$\begin{array}{l}{ }^{13} \mathrm{C} \text { formaldehyde } \\
(\mathrm{mM})\end{array}$} & \multirow{2}{*}{$\begin{array}{l}\text { Sodium azide } \\
(\mathrm{mM})\end{array}$} & \multirow{2}{*}{$\begin{array}{l}\text { Chlorhexidine } \\
(\mu \mathrm{M})\end{array}$} & \multirow{2}{*}{$\begin{array}{l}\text { Temperature } \\
\left({ }^{\circ} \mathrm{C}\right)\end{array}$} \\
\hline & & $\mathrm{CO}_{2}$ & ${ }^{13} \mathrm{CO}_{2}$ & & & & \\
\hline Autotrophy & Experimental & 0 & 20 & 0 & 0 & 0 & 65 \\
\hline Autotrophy & Experimental & 0 & 20 & 0 & 0.5 & 0 & 65 \\
\hline Autotrophy & Experimental & 0 & 20 & 0 & 0 & 63 & 65 \\
\hline Autotrophy & Control & 0 & 20 & 0 & 0 & 0 & 4 \\
\hline Formaldehyde uptake & Experimental & 20 & 0 & 3.2 & 0 & 0 & 65 \\
\hline Formaldehyde uptake & Experimental & 20 & 0 & 3.2 & 0.5 & 0 & 65 \\
\hline Formaldehyde uptake & Experimental & 20 & 0 & 3.2 & 0 & 63 & 65 \\
\hline Formaldehyde uptake & Control & 20 & 0 & 3.2 & 0 & 0 & 4 \\
\hline Formaldehyde uptake & Control & 20 & 0 & 3.2 & 0 & 0 & Autoclaved/65 \\
\hline
\end{tabular}

Incubations were designed to: (1) confirm that the sample handling and incubation protocols allowed the mat to continue autotrophic carbon fixation and assimilation, (2) determine whether formaldehyde was oxidized and/or converted to biomass within the mat, and (3) provide suitable controls to deconvolute biotic versus potential abiotic interactions between formaldehyde and the HFO mat and its microbial constituents. Quadruplicate samples were incubated under each of the conditions listed so that duplicate samples could be taken at two time points ( 3 and 7 days) (Table 1). Approximately $5 \mathrm{~mL}$ of a mat slurry (containing $\sim 0.5 \mathrm{~g}$ homogenized HFO mat in spring water) was dispensed into $125 \mathrm{~mL}$ glass bottles (with screw thread tops and each containing a septum, parts numbers 219439 and 240680, Wheaton Scientific Products, Millville, NJ) containing $25 \mathrm{~mL}$ of artificial spring water (see above) and prefilled with $0.2 \mathrm{~g}$ autoclaved pyrite. Once capped, bottles received different gaseous and liquid additions. All bottles received $20 \mathrm{~mL}$ of $\mathrm{CO}_{2}$, which was generated by addition of $\mathrm{HCl}$ to $\mathrm{NaHCO}_{3}$ containing either natural abundance $\mathrm{C}$ or enriched in ${ }^{13} \mathrm{C}$ (Cambridge Isotope Laboratories, Inc., Tewksbury, MA, USA). We added $0.25 \mathrm{~mL}$ of a $1 \%$ solution of ${ }^{13} \mathrm{C}$ labeled formaldehyde (99 atom $\%{ }^{13} \mathrm{C}$; SigmaAldrich, St. Louis, MO) to one set of treatments, and to different sets of bottles we also added either $0.25 \mathrm{~mL}$ of $0.5 \mathrm{M}$ sodium azide (Acros Organics, Thermo Fisher Scientific, Waltham MA, USA) or $1.0 \mathrm{~mL}$ of saturated chlorhexidine (1,1'-Hexamethylenebis[5-(4-chlorophenyl)biguanide]) (Sigma-Aldrich, St. Louis, MO, USA). We also performed a series of incubations using autoclaved (killed) mat as an additional control on the possible abiotic transformation and/or 'sorption' of formaldehyde to mat biomass. The autoclaved samples contained biomass harvested from the same spring, but from an earlier collection date (November 2013). All incubations were performed at either 65 or $4{ }^{\circ} \mathrm{C}$. Bottles were chilled upon harvesting and stored at $4{ }^{\circ} \mathrm{C}$ until analysis.

\section{Isotope ratio analysis-gas phase $\mathrm{CO}_{2}$ analysis}

Headspace was removed from each sample bottle and an acetone/dry ice trap was used to first remove water vapor from the sampled headspace and then a liquid nitrogen trap was used to freeze $\mathrm{CO}_{2}$ and allow pumping off all noncondensable gases. $\delta^{13} \mathrm{C}$ of the resulting purified $\mathrm{CO}_{2}$ was then measured in a dual inlet system on a Thermo Delta V Plus (Bremen, Germany) isotope ratio mass spectrometer (IRMS). Triplicate analytical measurements were performed for each biological duplicate. All ${ }^{13} \mathrm{C}$ stable isotope values are reported using delta $(\delta)$ notation where $\delta^{13} \mathrm{C}=\left(\mathrm{R}_{\mathrm{Sa}} / \mathrm{R}_{\mathrm{Std}}-1\right) \times 1000 \%$, and $\mathrm{R}_{\mathrm{Sa}}$ and $\mathrm{R}_{\mathrm{Std}}$ are the isotope ratio of the sample and intentionally recognized standard, respectively. The standard for $\mathrm{C}$ isotope analysis is Vienna Pee Dee Belemnite (VPDB). Note that only one of the biological duplicates of the $65^{\circ} \mathrm{C}, 7$-day incubation with chlorhexidine was measured for $\delta^{13} \mathrm{C}_{\mathrm{CO} 2}$ due to gas loss in sample handling.

\section{Isotope ratio analysis—solid phase/biomass analysis}

Biomass was harvested from the above incubations by centrifugation followed by treatment with an overnight acid wash $(1.2 \mathrm{~N} \mathrm{HCl}$ followed by multiple rinses with deionized water) to remove any inorganic carbon. Treated samples were lyophilized and homogenized in preparation for solid phase $\delta^{13} \mathrm{C}$ analysis. Once prepared, we weighed aliquots of each sample into tin capsules for $\delta^{13} \mathrm{C}$ analysis via a Costech Analytical Technologies (Valencia, CA, USA) 4010 Elemental Analyzer with a zero-blank autosampler coupled to a Thermo Delta V Plus IRMS. Data were corrected by the point-slope method (Coplen et al. 2006) using a series of in-house glutamic acid standards calibrated against the United State Geological Survey (USGS) 40 $\left(\delta^{13} \mathrm{C}=-26.39 \% \circ\right)$ and $41\left(\delta^{13} \mathrm{C}=+37.63 \%\right.$ international reference standards. We estimated the weight percent 
$\mathrm{C}$ of the analyzed samples by referencing resulting $\mathrm{CO}_{2}$ peak areas to those of an acetanilide standard (71.09 wt \% C).

\section{Genomic analysis}

Sequence assemblies corresponding to different phylotypes present in acidic HFO microbial mats of NGB were obtained from prior metagenome sequencing at Beowulf Spring and 100 Spring Plain (Inskeep et al. 2013; Inskeep et al. 2010). The genomic subassemblies analyzed were derived from iron oxide mats in YNP where organisms highly similar to M. yellowstonensis str. MK1 are found, including 100 Spring Plain (OSP_B, OSP_C, OSP_D, and OSP_14) and Beowulf Spring (BED) in the Norris Geyser Basin and Joseph's Coat Hot Springs (JCHS). In addition, genome sequences for YNP spring isolates, M. yellowstonensis MK1 and Sulfolobus islandicus YN.15.5.1 were also analyzed. IMG/ER (Markowitz et al. 2012, 2014b) and IMG/MER (Markowitz et al. 2014a) (http://jgi.doe.gov/data-and-tools/img/) were used to conduct searches for proteins assigned to KEGG orthology groups or containing functional domains expected to occur in enzymes responsible for formaldehyde metabolism.

\section{Results}

\section{Metallosphaera yellowstonensis MK1 formaldehyde analysis}

We grew pure cultures of $M$. yellowstonensis MK1 in artificial spring water using $\mathrm{CO}_{2}$ as the only carbon source for 14 days. NMR analysis of spent medium showed a peak which we preliminarily identified as formaldehyde located at a $\sim 3.214 \mathrm{ppm}$ shift (Fig. 1). This peak increased in size when we applied a formalin spike to the sample. The formalin used (Parasite-S, Western Chemical, Ferndale, WA) contained $37 \%$ formaldehyde with 6-14 \% methanol added as a chemical stabilizer. A Chenomx (Edmonton, Alberta, Canada) software package positively identified the methanol stabilizer at $\sim 3.365 \mathrm{ppm}$ leading us to conclude that the peak at $\sim 3.214 \mathrm{ppm}$, which aligned with that measured in the spent medium, was truly a formaldehyde signal. Increased signal intensity from a time zero to a mature culture sample identified formaldehyde as a metabolite secreted during autotrophic growth of $M$. yellowstonensis MK1. We used reference to the methanol peak (in the applied formalin) to estimate the formaldehyde concentration in the final culture medium to be $\sim 1 \mu \mathrm{M}$.

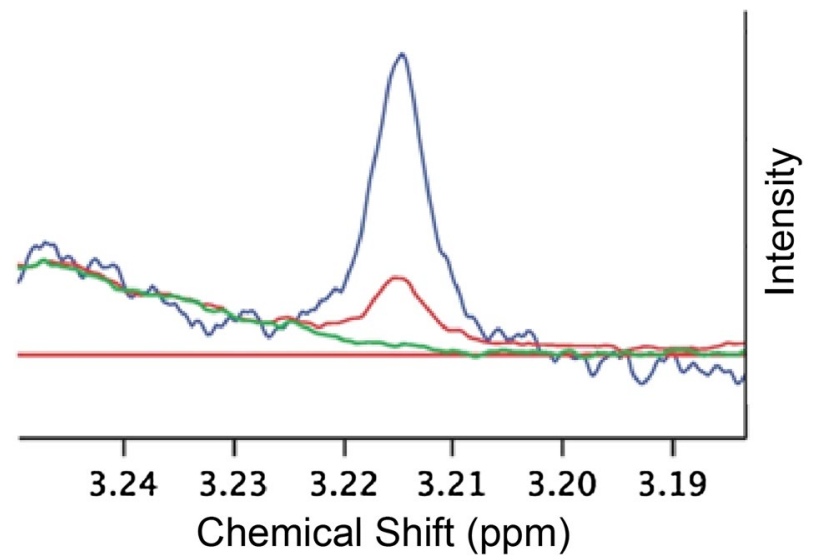

Fig. 1 NMR metabolite analysis. Proton NMR analysis of spent medium from $M$. yellowstonensis MK1 culture shows a formaldehyde-associated peak at a $\sim 3.214 \mathrm{ppm}$ shift (red). We used the formaldehyde peak resulting from a formalin spike (blue) to positively identify the formaldehyde peak and absence of this peak in the fresh medium (green) indicates formaldehyde production during culture growth. The flat, transverse line (red) represents the $x$ axis of the graph which intersects the $y$ axis at 0 . The measured spectral lines dip slightly below the $x$ axis (zero intensity on the right)

\section{$\delta^{13} \mathrm{C}$ analyses}

To determine the fate of formaldehyde in the HFO microbial community, we incubated ex situ samples of the microbial mat with ${ }^{13} \mathrm{C}$-formaldehyde and measured carbon isotope ratios in both the mat itself and in headspace $\mathrm{CO}_{2}$ after 3 and 7 days. Appearance of ${ }^{13} \mathrm{C}$ in biomass would indicate assimilation of formaldehyde-C, while appearance of ${ }^{13} \mathrm{C}$ in headspace $\mathrm{CO}_{2}$ would indicate oxidation. As positive tests for autotrophic activity, we incubated additional portions of the HFO mat with ${ }^{13} \mathrm{CO}_{2}$. As negative controls, we incubated samples of autoclaved mat.

HFO mat incubations with ${ }^{13} \mathrm{CO}_{2}$ showed strong uptake of $\mathrm{CO}_{2}$ into biomass when incubated at $65{ }^{\circ} \mathrm{C}$ (Fig. 2a, Supplementary Table 1), indicating active inorganic carbon fixation. Incubation at $4{ }^{\circ} \mathrm{C}$ or at $65{ }^{\circ} \mathrm{C}$ with added azide or chlorhexidine, however, showed essentially no autotrophic conversion of inorganic ${ }^{13} \mathrm{C}$ to biomass (Fig. 2a). The cessation of autotrophic activity in the $4{ }^{\circ} \mathrm{C}$ incubations was expected due to the large decrease in incubation temperature $\left(>60{ }^{\circ} \mathrm{C}\right)$ compared to the native hydrothermal system $\left(\sim 65{ }^{\circ} \mathrm{C}\right)$. Further, azide blocks the action of cytochrome oxidases within affected cells, while chlorhexidine damages cell membranes (McDonnell and Russell 1999; Winter et al. 2012). Based on these mechanisms of action, it is highly likely that the biocide additions did not specifically block autotrophy, but rather killed the autotrophic cells in the HFO mat. 

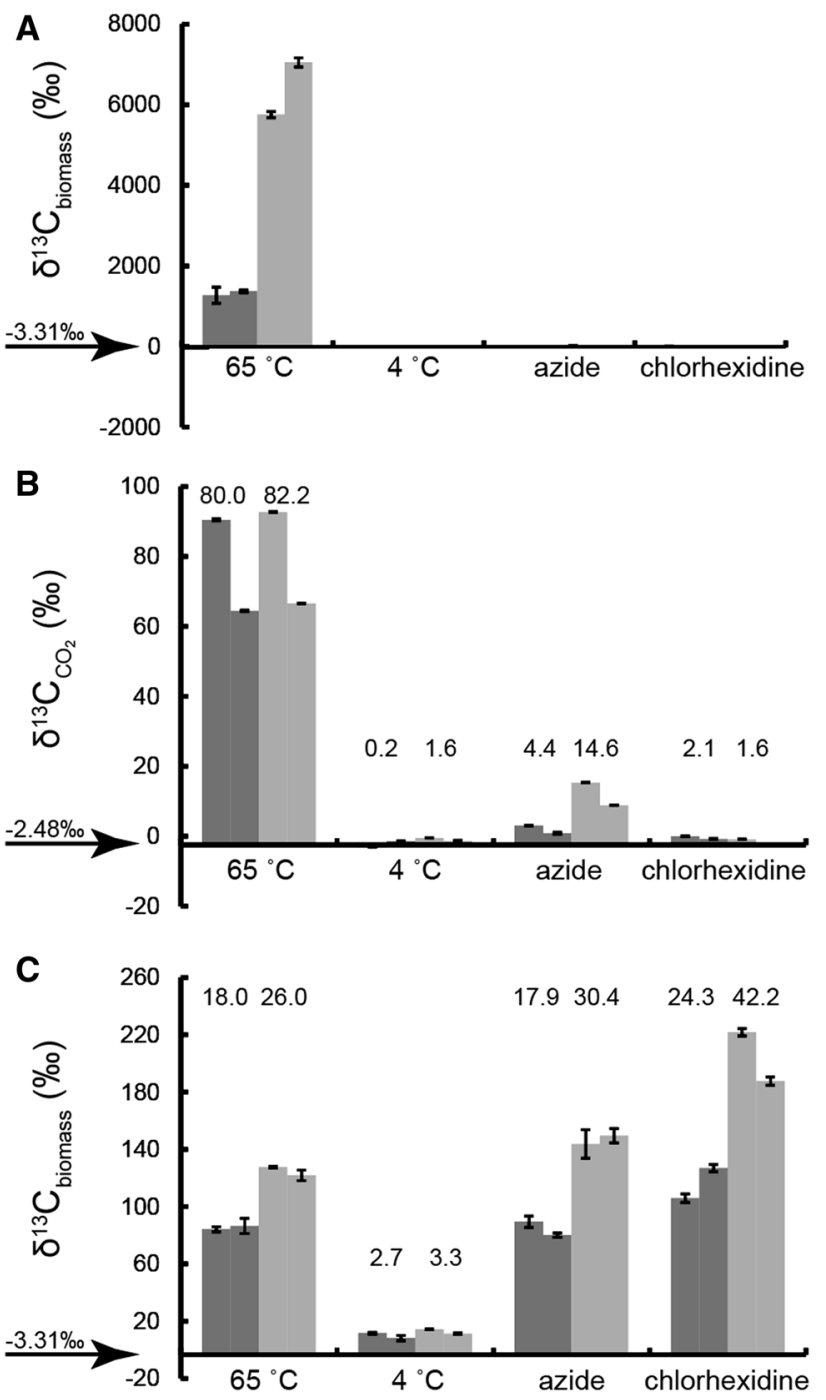

Fig. 2 Isotopic analysis of incubations. Measured $\delta^{13} \mathrm{C}$ of biomass (a, c) and headspace $\mathrm{CO}_{2}$ (b) after three (dark gray) and seven (light gray) days of incubation. a $\delta^{13} \mathrm{C}$ of incubation biomass above that of native mat $\left(-3.3 \%\right.$ ) reveals autotrophic conversion of ${ }^{13} \mathrm{C}$ labeled $\mathrm{CO}_{2}$ to biomass in the $65{ }^{\circ} \mathrm{C}$ incubations but vastly reduced autotrophy in the $4{ }^{\circ} \mathrm{C}$ incubation as well as the azide and chlorhexidine incubations (both at $65{ }^{\circ} \mathrm{C}$ ). b Oxidation of ${ }^{13} \mathrm{C}$ labeled formaldehyde to $\mathrm{CO}_{2}$ (enrichment above the starting $\delta^{13} \mathrm{C}_{\mathrm{CO} 2}=-2.5 \%$, estimated formaldehyde oxidation to $\mathrm{CO}_{2}$ noted above the data with the values representing nmoles of formaldehyde oxidized to $\mathrm{CO}_{2}$ ) is variable across the incubations but shows a sharp reduction in the $4{ }^{\circ} \mathrm{C}$ incubation as well as the azide and chlorhexidine incubations (both at $65{ }^{\circ} \mathrm{C}$ ) when compared to the $65^{\circ} \mathrm{C}$ incubation. c Formaldehyde assimilation to biomass (enrichment above the starting $\delta^{13} \mathrm{C}_{\text {biomass }}=-3.3 \%$, estimated formaldehyde assimilation to biomass noted above the data with values representing nmoles of formaldehyde assimilated into biomass) under all incubation conditions with the assimilation at $4{ }^{\circ} \mathrm{C}$ associated with abiotic formaldehyde binding to microbial biomass and all larger isotopic enrichments associated with microbial uptake. Error bars represent one standard deviation of analytical measurement replicates
Incubations of the HFO mat in the presence of ${ }^{13} \mathrm{C}$-formaldehyde showed both oxidation of formaldehyde to $\mathrm{CO}_{2}$ (Fig. 2b, S1) and assimilation of formaldehyde-derived carbon into HFO microbial biomass (Fig. 2c, S1). The initial $\delta^{13} \mathrm{C}$ value of the $\mathrm{CO}_{2}$ in the headspace of each formaldehyde incubation was $-2.5 \%$; we expected any increase in the ${ }^{13} \mathrm{C}$ content of final $\mathrm{CO}_{2}$ in an incubation to result from oxidation of ${ }^{13} \mathrm{C}$-formaldehyde into ${ }^{13} \mathrm{CO}_{2}$. To estimate the amount of formaldehyde oxidized to $\mathrm{CO}_{2}$ in each incubation, we assumed a constant amount of $\mathrm{CO}_{2}$ in each bottle $(20 \mathrm{~mL}, \mathrm{STP})$ then calculated the excess of ${ }^{13} \mathrm{C}$ (derived from labeled formaldehyde) required to increase the headspace $\delta^{13} \mathrm{C}_{\mathrm{CO} 2}$ from $-2.5 \%$ o to the measured value. Average estimates for formaldehyde oxidation in each set of biological replicates are reported (Fig. 2b) in units of nmoles formaldehyde oxidized and ranged from a low of 0.2 nmoles in the 3 -day $4{ }^{\circ} \mathrm{C}$ incubation to 82.2 nmoles in the 7-day $65{ }^{\circ} \mathrm{C}$ incubations. Incubating at $4{ }^{\circ} \mathrm{C}$ or at $65^{\circ} \mathrm{C}$ in the presence of azide or chlorhexidine significantly reduced formaldehyde oxidation. The incubations of autoclaved mat showed no oxidation of formaldehyde to $\mathrm{CO}_{2}$; instead these incubation showed a slight (statistically insignificant) decrease in the $\delta^{13} \mathrm{C}_{\mathrm{CO} 2}$ of $0.6 \%$ (Supplementary Table 1).

Formaldehyde-derived carbon was also incorporated into the HFO mat following incubation (Fig. 2c). The average $\delta^{13} \mathrm{C}$ of Beowulf Hot Spring HFO mat used for the incubations was $-3.3 \%$ and any increase of $\delta^{13} \mathrm{C}_{\text {biomass }}$ above $-3.3 \%$ would indicate conversion of applied ${ }^{13} \mathrm{C}$ label into microbial biomass in the ex situ incubations. All mat incubations showed increases in the amount of ${ }^{13} \mathrm{C}$ present when compared to the starting mat value, but with a large degree of variability between different incubation conditions. Incubations of autoclaved mat, used as a control for abiotic binding, showed an increase in $\delta^{13} \mathrm{C}$ of only $2.2 \%$ o compared to an average increase of $14.7 \%$ observed in the $4{ }^{\circ} \mathrm{C}$ incubations (when compared to the starting mat), showing that only a small portion of the observed $\delta^{13} \mathrm{C}$ enrichment could be attributed to abiotic binding of formaldehyde to biomass.

To estimate the amount of formaldehyde-C assimilated into biomass, we assumed a constant carbon mass in the mat and performed a mass balance analysis to calculate the amount of ${ }^{13} \mathrm{C}$ required to increase the amount of ${ }^{13} \mathrm{C}$ from the starting to the final, post-incubation measured biomass values. Elemental analysis indicated the final incubated mat contained $\sim 0.3 \% \mathrm{C}$ which, once adjustment was made for the added pyrite in the incubations, correlated to $\sim 0.6 \% \mathrm{C}$ for the pre-incubation HFO mat, a value similar to that reported by Jennings et al. (Jennings et al. 2014). 
Formaldehyde assimilation ranged from 2.7 nmoles in the 3-day incubation at $4{ }^{\circ} \mathrm{C}$ up to 42.2 nmoles in the seven day chlorhexidine incubation (Fig. 2c).

\section{Prediction of taxonomic distribution of formaldehyde metabolism}

The HFO community has been characterized by genomic analysis and previously described (Inskeep et al. 2013; Inskeep et al. 2010). The major phylogenetic groups present in these springs include M. yellowstonensis $(10-20 \%$ of the population based on $\%$ of total $16 \mathrm{~S}$ reads), other Sulfolobales including Sulfolobus (10-15\%), Novel Archaeal Group (NAG) 2 (up to $\sim 35 \%$ ), Geoarchaeota (formerly denoted NAG1) (up to $50 \%$ ), and Thermoproteales (up to $15 \%$ ). To explore which community members might be involved in formaldehyde production, assimilation, and oxidation, sequence assemblies corresponding to different phylotypes present in acidic HFO microbial mats of NGB, as well as genome sequences for YNP spring isolates $M$. yellowstonensis MK1 and Sulfolobus islandicus YN.15.5.1, were used to conduct searches for proteins assigned to KEGG orthology groups or containing functional domains expected to occur in enzymes responsible for formaldehyde metabolism.

The ribulose monophosphate pathway (RuMP) was found in the Sulfolobales order isolates (M. yellowstonensis MK1 and Sulfolobus islandicus YN.15.5.1), in NGB metagenome subassemblies from Sulfolobales-MK5 and Sulfolobales-Si, as well as in type 1 and 2 Thermoproteales order subassemblies obtained from a separate HFO spring (Joseph's Coat Spring). A complete set of enzymes required for the oxidative phase of the pentose phosphate pathway was not detected in any of the assemblies. The only potential formaldehyde oxidizing enzyme detected was the tungsten-dependent formaldehyde ferredoxin oxidoreductase (FOR), which was found in metagenome assemblies for novel archaeal group 2 (NAG2), Thermoproteales types 1 and 2, and Geoarchaeota NAG1.

\section{Discussion}

Formaldehyde production by pure M. yellowstonensis MK1 (Fig. 2) was not surprising given that formaldehyde is a common product of cellular demethylation reactions and is therefore almost ubiquitously produced in microbial systems. Formaldehyde production is typically highest, however, in methylotrophic communities where it is a key metabolic intermediate in central energy metabolism (Lidstrom 2006; Vorholt et al. 2000; Yurimoto et al. 2005) and is not expected to reach levels as high as we observed $(\sim 1 \mu \mathrm{M})$ solely as a byproduct of demethylation reactions.
Soderberg (2005) revealed that many archaeal species lack the full pentose phosphate pathway used to synthesize pentose sugars for subsequent anabolic processes (i.e. synthesis of nucleotides, histidine, and aromatic amino acids from pentose precursors). These organisms can employ a reverse RuMP pathway during which arabino-3-hexulose 6-phosphate (Hu6P) is converted to ribulose 5-phosphate (Ru5P) with the concurrent release of formaldehyde (Brasen et al. 2014; Orita et al. 2006).

Genome analysis of the members of this community revealed the presence of hallmark markers for this pathway (3-hexulose-6-phosphate synthase and 6-phospho3-hexuloisomerase), along with an absence of enzymes required for the oxidative phase of the pentose phosphate pathway in M. yellowstonensis MK-1. Flux balance analysis of Sulfolobus solfatarica, a close relative of M. yellowstonensis that also encodes the RuMP pathway and lacks enzymes required for the oxidative phase of the pentose phosphate pathway, showed that flux through reverse RuMP was necessary for production of nucleotides under autotrophic conditions (Ulas et al. 2012). Our observation of formaldehyde production during autotrophic growth by $\mathrm{M}$. yellowstonensis is consistent with this metabolic model.

M. yellowstonensis is the most abundant autotroph in the mature Beowulf HFO mat we sampled and likely contributes a significant proportion of its carbon from autotrophy (Jennings et al. 2014). Given the observation that autotrophically growing $M$. yellowstonensis releases formaldehyde into its environment, we sought to determine the fate of formaldehyde in the HFO community. Formaldehyde's inherent toxicity necessitates a removal process for maintaining viability in any microbial community producing the compound. Incubation of the sampled HFO mats in the presence of ${ }^{13} \mathrm{C}$-labeled formaldehyde highlighted two strategies for eliminating formaldehyde: oxidation to $\mathrm{CO}_{2}$ and assimilation into microbial biomass (Fig. 2b, c). Importantly, both of these conversions (to $\mathrm{CO}_{2}$ and to biomass) were drastically reduced in the $4{ }^{\circ} \mathrm{C}$ incubations and absent in incubations of autoclaved mat. The $4{ }^{\circ} \mathrm{C}$ incubations were $\sim 60{ }^{\circ} \mathrm{C}$ below the native $\mathrm{HFO}$ temperature and we applied this low temperature as a control for microbial activity. The small amount of formaldehyde 'assimilation' into microbial biomass observed at $4{ }^{\circ} \mathrm{C}$ could have occurred via abiotic binding of formaldehyde to amino groups within microbial biomass or as a result of greatly slowed metabolic activity, thereby representing an upper limit on abiotic binding. Taken together, the relatively high amounts of formaldehyde-derived $\mathrm{C}$ observed in both $\mathrm{CO}_{2}$ and biomass in the $65{ }^{\circ} \mathrm{C}$ incubations in contrast to the $4{ }^{\circ} \mathrm{C}$ and autoclaved mat incubations strongly suggest that both oxidation and assimilation are dominated by microbial processes. 
Table 2 Phylogenetic distribution of metabolic pathways for and biocide sensitivity of production, oxidation, and assimilation of formaldehyde in HFO communities

\begin{tabular}{|c|c|c|c|c|}
\hline Function & Gene or pathway & Phylogenetic group & Response to azide $^{\mathrm{a}}$ & Response to chlorhexidine ${ }^{a}$ \\
\hline $\begin{array}{l}\text { Formaldehyde production } \\
\text { via autotrophy }\end{array}$ & $\begin{array}{l}\text { 3-hydroxypropionate } \\
\text { 4-hydroxybutyrate C } \\
\text { fixation pathway, Reverse } \\
\text { RuMP }\end{array}$ & $\begin{array}{l}\text { M. yellowstonensis, Pos- } \\
\text { sibly other Sulfolobales }\end{array}$ & Inactivated & Inactivated \\
\hline Formaldehyde oxidation & $\begin{array}{l}\text { Tungsten-dependent } \\
\text { formaldehyde ferredoxin } \\
\text { oxidoreductase }\end{array}$ & $\begin{array}{l}\text { Geoarchaeota (NAG1), } \\
\text { NAG2, Thermoproteales }\end{array}$ & Reduced & $\begin{array}{l}\text { Greatly reduced but detect- } \\
\text { able }\end{array}$ \\
\hline Formaldehyde assimilation & RuMP & $\begin{array}{l}\text { M. yellowstonensis, pos- } \\
\text { sibly other Sulfolobales, } \\
\text { Thermoproteales }\end{array}$ & Essentially unchanged & Increased \\
\hline
\end{tabular}

${ }^{\text {a }}$ Compared to activity at $65^{\circ} \mathrm{C}$

Formaldehyde assimilation into biomass proceeds via two known enzymatic pathways in prokaryote systems, the serine and the ribulose monophosphate pathways (RuMP) (Kemp and Quayle 1966). Metagenomic analysis specifically identified the RuMP pathway attributable to two different novel archaeal genomes falling within the Thermoproteales order and composing 10-15\% of the biomass collected at a nearby HFO spring (100 Spring Plain) with similar geochemical conditions (Inskeep et al. 2013; Kozubal et al. 2012), as well as in the Sulfolobales assemblies from the spring community and the genomes of M. yellowstonensis MK1 and Sulfolobus islandicus YN.15.5.1. Sequences associated with the serine pathway were not detected. The two reactions of the RuMP pathway are reversible; the kinetics of isolated enzymes are more favorable in the forward reaction (Orita et al. 2006), with the reverse direction thought to be driven by continuous consumption of ribulose-5-phosphate for nucleotide biosynthesis. Our incubation conditions supplied $\mathrm{CO}_{2}$ in all the experiments-labeled $\mathrm{CO}_{2}$ in the experiments shown in $2 \mathrm{a}$, and unlabeled $\mathrm{CO}_{2}$ in Fig. $2 \mathrm{~b}$, c. Presumably, then, the RuMP pathway in M. yellowstonensis and any other autotrophic Sulfolobales in the community was operating at least largely in reverse in incubations at $65{ }^{\circ} \mathrm{C}$ and in the absence of inhibitors, though those organisms might be incorporating a small amount of formaldehyde.

We applied two different biocides, azide and chlorhexidine, to the incubations to test their effect on carbon fixation, formaldehyde oxidation and assimilation. Azide binds to heme-iron (including cytochrome oxidase and catalase) and can cause cellular chemical asphyxiation (McDonnell and Russell 1999; Winter et al. 2012). Azide is generally considered more effective at limiting the growth of aerobic versus anaerobic organisms (Forget and Fredette 1962). Chlorhexidine causes membrane disruption in susceptible organisms (McDonnell and Russell 1999) and, while it is considered a broad spectrum biocide, chlorhexidine is not universally effective (Mangram et al. 1999; Russell 1986). Given the functional targets of azide (heme-iron) and chlorhexidine (membrane disruption) it is unlikely these biocides targeted only specific metabolic pathways but, rather that they inactivated the entire affected cells. The effects of the biocides on metabolic processes affecting formaldehyde, along with the responsible gene or pathway, and the phylogenetic groups within the community associated with the pathways, are summarized in Table 2.

One potential explanation for assimilation of formaldehyde- $\mathrm{C}$ into biomass is that the formaldehyde was first oxidized, and then the resulting ${ }^{13} \mathrm{CO}_{2}$ was assimilated into biomass via autotrophic carbon fixation. However, the addition of azide or chlorhexidine inactivated the autotrophs without reducing assimilation (Fig. 2c). The continuation of formaldehyde assimilation following collapse of autotrophic activity in both the azide and chlorhexidine incubations demonstrates that (1) assimilation of formaldehyde- $\mathrm{C}$ does not proceed via oxidation and then $\mathrm{C}$ fixation, and (2) the autotrophic population of the HFO mat, dominated by $M$. yellowstonensis, is not the primary formaldehyde consumer. The portion of the community with the genetic potential (via the RuMP pathway, Table 1) to take up formaldehyde in the presence of inhibitors consists of the Thermoproteales and, presumably, non-autotrophic Sulfolobales.

In contrast to the case for formaldehyde assimilation, exposure to either azide or chlorhexidine significantly reduced formaldehyde assimilation versus the $65{ }^{\circ} \mathrm{C}$ incubations (Fig. 2b). The extent of formaldehyde oxidation in the chlorhexidine incubation mirrored the low levels in the $4{ }^{\circ} \mathrm{C}$ incubation. Application of azide reduced the amount of formaldehyde oxidation compared to the $65{ }^{\circ} \mathrm{C}$ incubation but significantly more activity remained than in the chlorhexidine incubations. Formaldehyde oxidation can be enzymatically performed by formaldehyde ferredoxin oxidoreductases (FORs), and metagenomic analysis of Norris 


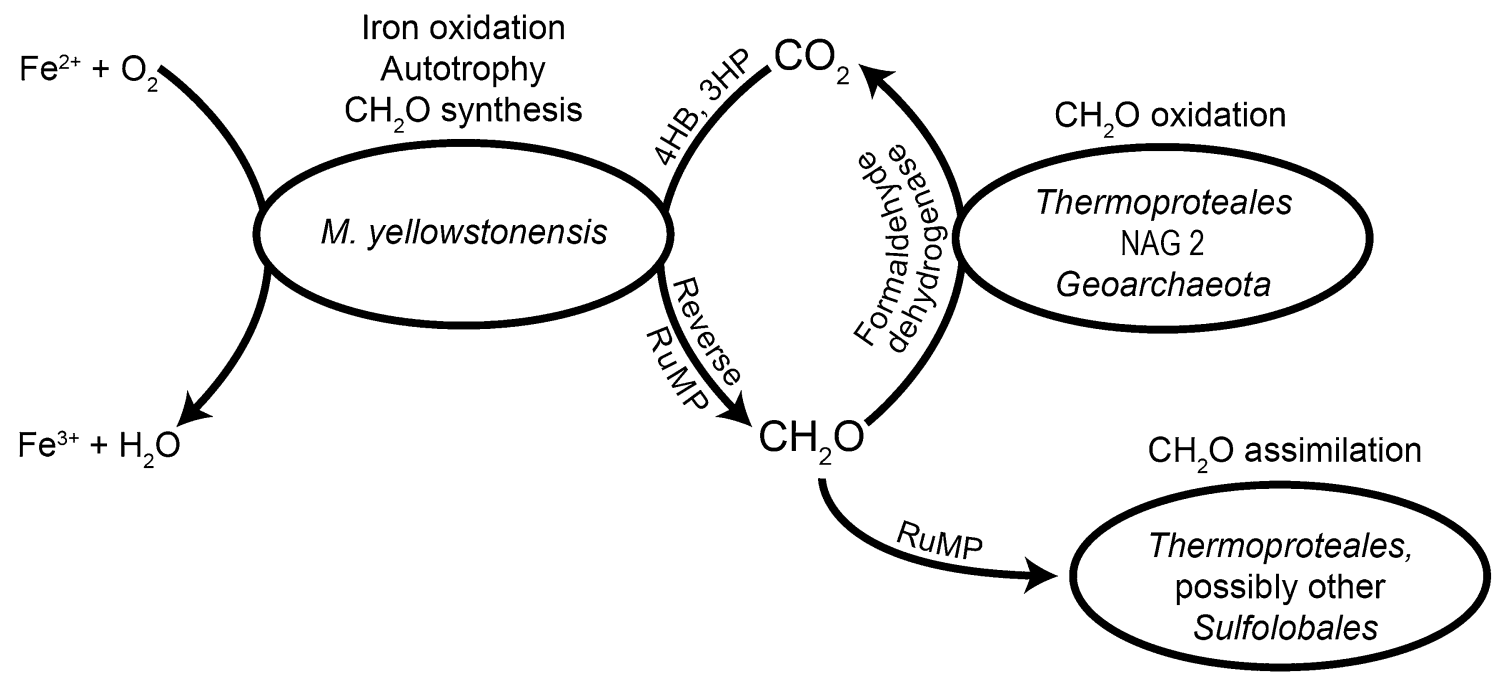

Fig. 3 Formaldehyde as a carbon and electron shuttle between populations in the hydrous ferric oxide microbial mat. The suite of incubations identified three formaldehyde-related populations, those associated with formaldehyde production, oxidation, and consumption. The formaldehyde producer (M. yellowstonensis and possibly other autotrophic Sulfolobales constituting a minor component of the mat community) fixes $\mathrm{C}$ using electrons derived from iron oxidation via

Geyser Basin HFO mats identified FOR sequences linked to organisms belonging to a novel archaeal group (NAG2), the Thermoproteales order, and the Geoarchaeota phylum (Kozubal et al. 2012, 2013). The reduced, but continued, oxidation of formaldehyde in the presence of azide is consistent with multiple community members contributing to formaldehyde oxidation, some of which are azide-resistant.

Iron oxidation and its associated autotrophy provide the primary energy and carbon inputs to the HFO mats. Since $M$. yellowstonensis lacks a complete oxidative pentose phosphate pathway, it is an obligatory formaldehyde producer (via the reverse RuMP pathway) for a number of anabolic synthesis reactions. The reverse RuMP pathway can produce relatively high concentrations of formaldehyde which, due to its inherent toxicity, must be removed to maintain overall community viability (Brasen et al. 2014). The variable responses of autotrophy, formaldehyde oxidation, and formaldehyde assimilation to azide and chlorhexidine suggest that three distinct sub-populations of organisms participate in formaldehyde cycling within the HFO mats: formaldehyde producers, oxidizers, and assimilators (Fig. 3). Given the diversity of organisms involved with formaldehyde cycling in this HFO mat, the formaldehyde consumption is likely controlled by the larger microbial community instead of simply by the producing organism. The excreted formaldehyde can function as a shuttle whereby a portion of the energy and carbon first fixed via iron oxidation and autotrophy are passed to associated heterotrophic organisms. These heterotrophs, by consuming the formaldehyde the 4-hydroxybutyrate/3-hydroxypropionate pathway and excretes formaldehyde produced by the reverse RuMP pathway. Resulting formaldehyde is assimilated via the forward RuMP by Thermoproteales and/or non-autotrophic Sulfolobales and oxidized to $\mathrm{CO}_{2}$ (via aldehyde ferredoxin oxidoreductase) by another, albeit not mutually exclusive, group within the community that includes Geoarchaeota, NAG1, and Thermoproteales)

as a carbon and energy source, reduce any toxic effects of its production on the system's primary producer.

Acknowledgments This research was supported by the Genomic Science Program (GSP), Office of Biological and Environmental Research (OBER), U.S. Department of Energy (DOE), and is a contribution of the Pacific Northwest National Laboratory (PNNL) Foundational Scientific Focus Area. A portion of the research was performed using EMSL, a DOE Office of Science User Facility sponsored by the Office of Biological and Environmental Research and located at Pacific Northwest National Laboratory. A portion of the research was performed by the Joint Genome Institute (DE-AC02-05CH11231) supported by the Genome Science Program, Office of Biological and Environmental Research, US DOE through a Community Sequencing Project to WPI (CSP 787081). We appreciate research permit (permit no. YELL-2014-SCI-5068) and helpful discussions and assistance with sample collection from J. Beam, H. Bernstein, K. Dana, Z. Jay, R. Jennings, M. Kozubal, and Y. Maezato.

\section{References}

Beam JP, Jay ZJ, Kozubal MA, Inskeep WP (2014) Niche specialization of novel Thaumarchaeota to oxic and hypoxic acidic geothermal springs of Yellowstone National Park. ISME J 8:938951. doi:10.1038/ismej.2013.193

Boyd ES, Leavitt WD, Geesey GG (2009) CO2 uptake and fixation by a thermoacidophilic microbial community attached to precipitated sulfur in a geothermal spring. Appl Environ Microbiol 75:4289-4296. doi:10.1128/aem.02751-08

Brasen C, Esser D, Rauch B, Siebers B (2014) Carbohydrate metabolism in Archaea: current insights into unusual enzymes and pathways and their regulation. Microbiol Mol Biol R 78:89-175. doi:10.1128/Mmbr.00041-13 
Coplen TB, Brand WA, Gehre M, Groning M, Meijer HAJ, Toman B, Verkouteren RM (2006) New guidelines for delta C-13 measurements. Anal Chem 78:2439-2441. doi:10.1021/Ac052027c

Forget A, Fredette V (1962) Sodium azide selective medium for primary isolation of anaerobic bacteria. J Bacteriol 83:1217

Inskeep WP, Macur RE, Harrison G, Bostick BC, Fendorf S (2004) Biomineralization of $\mathrm{As}(\mathrm{V})$-hydrous ferric oxyhydroxide in microbial mats of an acid-sulfate-chloride geothermal spring, Yellowstone National Park. Geochim Cosmochim Acta 68:31413155. doi:10.1016/j.gca.2003.09.020

Inskeep WP, Ackerman GG, Taylor WP, Kozubal M, Korf S, Macur RE (2005) On the energetics of chemolithotrophy in nonequilibrium systems: case studies of geothermal springs in Yellowstone National Park. Geobiology 3:297-317. doi:10.1111/j.1472-4669.2006.00059.x

Inskeep WP et al (2010) Metagenomes from high-temperature chemotrophic systems reveal geochemical controls on microbial community structure and function. PLoS One. doi:10.1371/journal. pone.0009773

Inskeep WP et al (2013) Phylogenetic and functional analysis of metagenome sequence from high-temperature archaeal habitats demonstrate linkages between metabolic potential and geochemistry. Front Microbiol. doi:10.3389/Fmicb.2013.00095

Jennings RM, Whitmore LM, Moran JJ, Kreuzer HW, Inskeep WP (2014) Carbon Dioxide Fixation by Metallosphaera yellowstonensis and Acidothermophilic Iron-Oxidizing Microbial Communities from Yellowstone National Park. Appl Environ Microb 80:2665-2671. doi:10.1128/Aem.03416-13

Kemp MB, Quayle JR (1966) Microbial growth on C1 compoundsincorporation of $\mathrm{C} 1$ units into allulose phosphate by extracts of pseudomonas methanica. Biochem J 99:41

Kozubal M, Macur RE, Korf S, Taylor WP, Ackerman GG, Nagy A, Inskeep WP (2008) Isolation and distribution of a novel ironoxidizing crenarchaeon from acidic geothermal springs in Yellowstone National Park. Appl Environ Microbiol 74:942-949. doi:10.1128/aem.01200-07

Kozubal MA et al (2012) Microbial iron cycling in acidic geothermal springs of Yellowstone National Park: integrating molecular surveys, geochemical processes, and isolation of novel Fe-active microorganisms. Front Microbiol. doi:10.3389/ Fmicb.2012.00109

Kozubal MA et al (2013) Geoarchaeota: a new candidate phylum in the Archaea from high-temperature acidic iron mats in Yellowstone National Park. ISME J 7:622-634. doi:10.1038/ ismej.2012.132

Langner HW, Jackson CR, Mcdermott TR, Inskeep WP (2001) Rapid oxidation of arsenite in a hot spring ecosystem, Yellowstone National Park. Environ Sci Technol 35:3302-3309. doi:10.1021/ Es0105562
Lidstrom ME (2006) Aerobic methylotrophic procaryotes. In: Balows A., Truper HG, Dworkin M, Harder W, Schleifer KH (eds) The Prokaryotes. Springer, New York, pp 613-634

Mangram AJ, Horan TC, Pearson ML, Silver LC, Jarvis WR (1999) Guideline for prevention of surgical site infection. Infect Control Hosp Epidemiol 20:250-278. doi:10.1086/501620

Markowitz VM et al (2012) IMG: the integrated microbial genomes database and comparative analysis system. Nucleic Acids Res 40:D115-D122. doi:10.1093/Nar/Gkr1044

Markowitz VM et al (2014a) IMG/M 4 version of the integrated metagenome comparative analysis system. Nucleic Acids Res 42:D568-D573. doi:10.1093/Nar/Gkt919

Markowitz VM et al (2014b) IMG 4 version of the integrated microbial genomes comparative analysis system. Nucleic Acids Res 42:D560-D567. doi:10.1093/Nar/Gkt963

McDonnell G, Russell AD (1999) Antiseptics and disinfectants: activity, action, and resistance. Clin Microbiol Rev 12:147

Orita I, Sato T, Yurimoto H, Kato N, Atomi H, Imanaka T, Sakai Y (2006) The ribulose monophosphate pathway substitutes for the missing pentose phosphate pathway in the archaeon Thermococcus kodakaraensis. J Bacteriol 188:4698-4704. doi:10.1128/ $\mathrm{Jb} .00492 .06$

Russell AD (1986) Chlorhexidine-antibacterial action and bacterialresistance infection 14:212-215. doi:10.1007/Bf01644264

Singer PC, Stumm W (1970) Acidic mine drainage. Rate-determining step. Science 167:1121. doi:10.1126/science.167.3921.1121

Soderberg T (2005) Biosynthesis of ribose-5-phosphate and erythrose-4-phosphate in archaea: a phylogenetic analysis of archaeal genomes. Archaea 1:347-352

Ulas T, Riemer SA, Zaparty M, Siebers B, Schomburg D (2012) Genome-scale reconstruction and analysis of the metabolic network in the hyperthermophilic archaeon sulfolobus solfataricus. PLoS One. doi:10.1371/journal.pone.0043401

Vorholt JA, Marx CJ, Lidstrom ME, Thauer RK (2000) Novel formaldehyde-activating enzyme in methylobacterium extorquens AM1 required for growth on methanol. J Bacteriol 182:6645-6650. doi:10.1128/Jb.182.23.6645-6650.2000

Winter C, Kerros ME, Weinbauer MG (2012) Effects of sodium azide on the abundance of prokaryotes and viruses in marine samples. PLoS One. doi:10.1371/journal.pone.0037597

Yurimoto H, Kato N, Sakai Y (2005) Assimilation, dissimilation, and detoxification of formaldehyde, a central metabolic intermediate of methylotrophic metabolism. Chem Rec 5:367-375. doi:10.1002/Tcr.20056 